\title{
Highly interconnected genes in disease-specific networks are enriched for disease-associated polymorphisms
}

\author{
Fredrik Barrenäs ${ }^{1 \dagger}$, Sreenivas Chavali ${ }^{2 \dagger}$, Alexessander Couto Alves ${ }^{3}$, Lachlan Coin ${ }^{3}$, Marjo-Riitta Jarvelin ${ }^{3,4}$, \\ Rebecka Jörnsten ${ }^{5}$, Michael A Langston ${ }^{6}$, Adaikalavan Ramasamy ${ }^{3,7}$, Gary Rogers ${ }^{6}$, Hui Wang ${ }^{1}$ and \\ Mikael Benson ${ }^{1,8^{*}}$
}

\begin{abstract}
Background: Complex diseases are associated with altered interactions between thousands of genes. We developed a novel method to identify and prioritize disease genes, which was generally applicable to complex diseases.

Results: We identified modules of highly interconnected genes in disease-specific networks derived from integrating gene-expression and protein interaction data. We examined if those modules were enriched for disease-associated SNPs, and could be used to find novel genes for functional studies. First, we analyzed publicly available gene expression microarray and genome-wide association study (GWAS) data from 13, highly diverse, complex diseases. In each disease, highly interconnected genes formed modules, which were significantly enriched for genes harboring disease-associated SNPs. To test if such modules could be used to find novel genes for functional studies, we repeated the analyses using our own gene expression microarray and GWAS data from seasonal allergic rhinitis. We identified a novel gene, FGF2, whose relevance was supported by functional studies using combined small interfering RNA-mediated knock-down and gene expression microarrays. The modules in the 13 complex diseases analyzed here tended to overlap and were enriched for pathways related to oncological, metabolic and inflammatory diseases. This suggested that this union of the modules would be associated with a general increase in susceptibility for complex diseases. Indeed, we found that this union was enriched with GWAS genes for 145 other complex diseases.
\end{abstract}

Conclusions: Modules of highly interconnected complex disease genes were enriched for disease-associated SNPS, and could be used to find novel genes for functional studies.

\section{Background}

Medical research often focuses on individual diseases and genes. However, complex diseases show considerable comorbidity and are associated with altered interactions between thousands of genes. This suggests a need to find generally applicable principles to study multiple diseases and genes. One solution may be to map differentially expressed, disease-associated genes on to the human protein-protein interaction (PPI) network. Gene expression

\footnotetext{
* Correspondence: mikael.benson@liu.se

+ Contributed equally

'The Centre for Individualized Medication, Linköping University Hospital, Linköping University, Linköping, SE-58185, Sweden

Full list of author information is available at the end of the article
}

microarray studies of several complex diseases have shown that differentially expressed genes tend to form modules of interacting and functionally related genes [1-5]. Those modules may help to identify genes harboring diseaseassociated SNPs [6]. The identification is, however, complicated by the involvement of multiple modules in the same complex disease.

In this study, we hypothesized that modules containing the most interconnected complex disease-associated genes would be enriched for disease-associated SNPs (note that highly interconnected disease genes have many interactions with other disease genes, while hub genes have interactions with any other gene). This hypothesis was based on recent discoveries in network medicine. 
Firstly, the effects of disease-associated SNPs tend to propagate through the PPI network, affecting the local neighborhood around the SNP-harboring genes [7-9]. Secondly, genes harboring disease-associated SNPs tend to form modules in the PPI network [10-13]. Those studies are mainly based on rare hereditary diseases. A recent meta-analysis of genome-wide association studies (GWAS), however, suggests that genes harboring diseaseassociated SNPs in complex diseases are also highly interconnected [14]. Taken together, previous studies showed that genes harboring disease-associated SNPs tend to form modules, and that the same is true for differentially expressed genes [15]. In this study, we integrated these two findings and used modules formed by differentially expressed disease genes to find genes harboring diseaseassociated SNPs. For this, first we defined modules in disease-specific networks for 13 complex diseases and show that these highly interconnected genes in these modules are enriched for disease genes identified through GWAS. To test the general applicability of our findings, the selected diseases were highly diverse and included oncological, metabolic and inflammatory diseases. Using inhouse generated gene-expression and GWAS data, we showed that such modules could be used for identifying novel genes for functional studies, using seasonal allergic rhinitis (SAR) as a disease model. Finally, we show that overlapping modules of the complex diseases are generally enriched for genes harboring disease-associated SNPs, especially pleiotropic genes, identified by GWAS of 145 complex diseases.

\section{Results}

Disease-specific core susceptibility modules are enriched for disease-associated genes in 13 complex diseases

We defined modules in disease-specific networks by using (a) the global human PPI network and (b) differentially expressed genes for each disease. Those modules will henceforth be referred to as susceptibility modules (SuMs). Genes with high interconnectivity in the SuMs were defined as core SuMs (Figure 1; see Materials and methods). We examined whether the disease-specific core SuMs were enriched for genes harboring diseaseassociated SNPs by analyzing complex diseases for which gene expression microarray data from relevant cells or tissues were available in the public domain, and where GWAS had identified genes harboring disease-associated SNPs. Such genes will henceforth be referred to as GWAS genes. Thirteen oncological, immunological or metabolic diseases fulfilled these criteria (Table 1; Additional file 1). For each disease, we derived a SuM and within it, a core SuM. The enrichment of GWAS genes in the core SuMs was 4.71-fold compared to the whole PPI network $\left(P<10^{-5}\right)$. The corresponding figure of the SuMs was 2.22 -fold $\left(P<10^{-5}\right)$. In contrast, using only differentially expressed genes we found a mere 1.15-fold enrichment of GWAS genes $(P=0.3$; Figure $2 \mathrm{a})$. We tested different cutoffs for interconnectivity. We found that increasingly stringent cutoffs for core SuMs were associated with stronger enrichment of GWAS genes (Figure 2b). Based on these analyses we defined core SuMs as the $10 \%$ of the SuM genes with the lowest average shortest path length. This demonstrated the effectiveness of modules to identify GWAS genes, compared to differentially expressed genes.

\section{Core SuMs could be used to find novel genes for functional studies}

We then determined whether core SuMs could be used to find novel genes for functional studies. We repeated the analyses using our own gene expression microarray and GWAS data from patients with SAR. This is an ideal model of complex diseases because it is possible to mimic and analyze the disease process in allergen-challenged cells from patients [16] (Extended background in Additional file 2). Novel genes can be functionally examined by combining small interfering RNA (siRNA) and gene expression microarrays in Th2 polarized cells [17-19], (Extended background in Additional file 2).

We constructed a SuM and a core SuM for SAR using differentially expressed genes obtained by performing gene expression microarray analysis of allergen-challenged CD4+ cells in samples from 12 patients as previously described $[19,20]$ (Additional file 3 ). The SuM included 622 of the 2,822 differentially expressed genes in the PPI network, and 1,191 genes in total (Figure 1a in Additional file 4). Next, we tested if they could be replicated in the additional study material. The repeated analyses resulted in highly similar SuMs and core SuMs $(P<$ $10^{-15}$ in both cases, determined by a $\chi^{2}$-test; Figure $1 \mathrm{~b}$ in Additional file 4). We also compared gene expression microarray data from allergen-challenged CD4+ cells from patients with SAR and allergen-challenged CD4+ cells from healthy controls and found differences in disease-relevant pathways and genes (Extended results in Additional file 2).

To determine whether the genes in the SuM and core SuM were enriched for SAR-associated SNPs, we analyzed an independent GWAS of 4,772 individuals in the North Finland Birth Cohort (Figure 1e). We found that intragenic SNPs within the 119 genes in the core SuM were 3.4 times more likely to be disease-associated than expected by chance $\left(P=1 \times 10^{-5}\right)$. This led to the identification of two novel genes, FGF2 and MAPK8 (Additional file 5). These findings were appropriately supported by false discovery rate calculations (Extended results in Additional file 2; Additional file 6). These genes had not been previously associated with SAR [21]. While $M A P K 8$ has a known role in type 1 allergic inflammation [22-24], FGF2 is a novel 

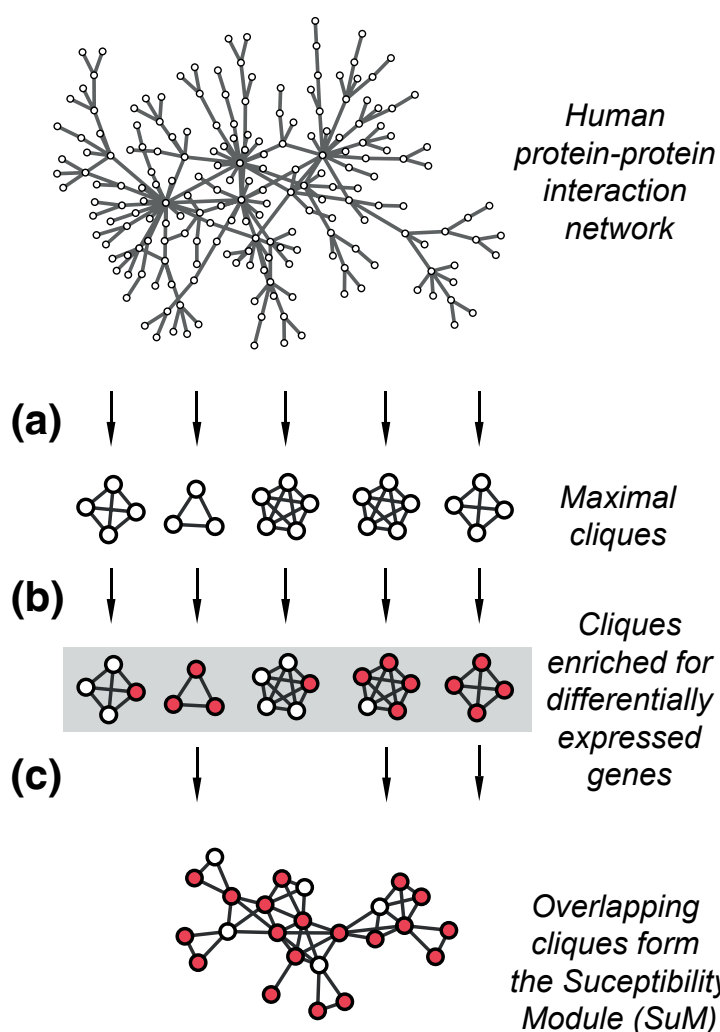

Overlapping cliques form the Suceptibility Module (SuM)

(d)

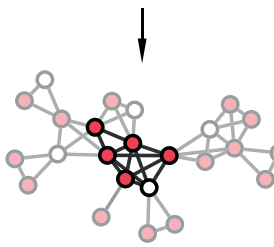

The most interconnected genes form the core SuM

(e)

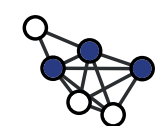

\section{Enrichment of GWAS genes in core SuM}

Figure 1 Overview of identification of susceptibility modules (SuMs). (a) Maximal cliques were obtained from a human PPI. (b) Diseaseassociated cliques were identified by selecting those that were enriched for differentially expressed genes. (c) Such cliques were mapped onto the PPI network, resulting in the identification of a SuM of overlapping cliques. (d) A core SuM was identified using average shortest path length. (e) This core SuM was validated by showing enrichment for GWAS genes.

gene. We tested the functional relevance of FGF2 by siRNA-mediated knock-down of this gene in Th2 polarized cells, followed by gene expression microarrays (Extended methods and Extended results in Additional file 2; Additional file 7). The knock-down resulted in altered expression of several pathways of potential relevance for type 1 allergic inflammation, as well as individual genes of known relevance for type 1 allergic inflammation, including MAFB and NFKB1 [25-30] (Additional file 8). In contrast to the core SuM, no enrichment of SNPs was found in the SuM.
The union of core SuMs of different diseases was enriched for pleiotropic genes

Because complex diseases tend to show both phenotypic and genotypic overlap [31,32], we hypothesized that the core SuMs would be associated with generally increased susceptibility for all complex diseases. In support of this assertion, we found that the core SuMs from the 13 diseases tended to overlap. This tendency was stronger for core SuMs than SuMs (Figure 3a). The union of the core SuMs was highly enriched for pathways involved in oncological, metabolic and inflammatory complex 
Table 1 Overview of SuMs in 13 complex diseases

\begin{tabular}{|c|c|c|c|c|c|c|c|}
\hline \multirow[b]{2}{*}{ Disease } & \multirow[b]{2}{*}{ GEO accession } & \multirow[b]{2}{*}{ Genes in SuM } & \multirow{2}{*}{$\begin{array}{c}\text { GWAS genes in PPI } \\
\text { network }\end{array}$} & \multicolumn{2}{|c|}{ GWAS genes in SuM } & \multicolumn{2}{|c|}{ GWAS genes in core SuM } \\
\hline & & & & Observed & Expected & Observed & Expected \\
\hline Asthma & GSE4302 & 587 & 8 & 1 & 0.39 & 0 & 0.04 \\
\hline Breast cancer & GSE10810 & 2,474 & 7 & 2 & 1.45 & 1 & 0.15 \\
\hline Chronic lymphocytic leukemia & GSE8835 & 1,787 & 11 & 3 & 1.64 & 0 & 0.17 \\
\hline Colorectal cancer & GSE9348 & 1,651 & 5 & 3 & 0.69 & 1 & 0.07 \\
\hline Crohn's disease & GSE6731 & 1,458 & 16 & 5 & 1.95 & 3 & 0.20 \\
\hline Lung adenocarcinoma & GSE7670 & 2,524 & 10 & 4 & 2.11 & 0 & 0.21 \\
\hline Obesity & GSE12050 & 2,268 & 13 & 4 & 2.47 & 0 & 0.25 \\
\hline Parkinson's disease & GSE20141 & 1,871 & 21 & 5 & 3.29 & 1 & 0.33 \\
\hline Prostate cancer & GSE6919 & 751 & 16 & 3 & 1.01 & 1 & 0.10 \\
\hline Psoriasis & GSE13355 & 2,274 & 12 & 8 & 2.28 & 1 & 0.23 \\
\hline Schizophrenia & GSE17612 & 1,586 & 18 & 4 & 2.39 & 0 & 0.24 \\
\hline Type 2 diabetes & GSE20966 & 1,658 & 21 & 5 & 2.91 & 2 & 0.29 \\
\hline Ulcerative colitis & GSE6731 & 2,365 & 25 & 13 & 4.95 & 3 & 0.50 \\
\hline Total & - & - & - & 60 & 27.53 & 13 & 2.76 \\
\hline
\end{tabular}

Gene expression dataset for each disease obtained from the NCBI Gene Expression Omnibus (GEO).

diseases (Additional file 9). Finally, we tested whether the union of the core SuMs was generally enriched for GWAS genes of various complex diseases. This test comprised 1,570 GWAS genes associated with 145 complex diseases, excluding the genes associated with the 13 complex diseases for which the core SuMs were derived (Additional file 1). We found 2.52-fold enrichment
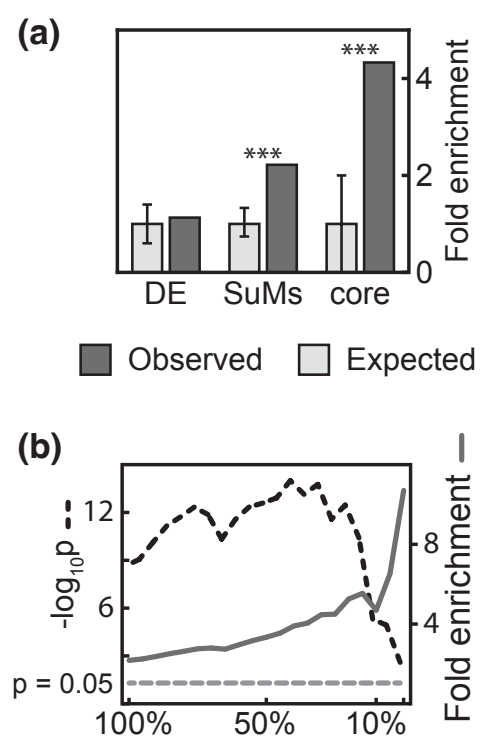

Figure 2 Enrichment of GWAS genes from 13 oncological, immunological and metabolic diseases in SuMs and core SuMs. (a) The enrichment of GWAS genes in core SuMs was stronger when the cutoff was more restrictive. The $10 \%$ cutoff was chosen to define core SuMs. (b) SuMs and core SuMs show strong enrichment of GWAS genes compared to differentially expressed (DE) genes. Error bars represent the $95 \%$ confidence interval of the randomized selections. Asterisks represent $\mathrm{p}<0.001$. compared to the whole PPI network $\left(P<10^{-18}\right)$. The enrichment increased if the disease genes were pleiotropic, that is, associated with more than one disease (Figure $3 \mathrm{~b}$ ). For example, when considering only genes associated with more than one disease, we found a 3.1fold enrichment $\left(P<10^{-6}\right)$, and when considering genes only associated with more than four diseases we found a 9.1-fold enrichment $\left(P<10^{-3}\right)$.

\section{Discussion}

We have developed a novel method to define SuMs and core SuMs for complex diseases by combining geneexpression microarray and PPI data. To show the general applicability of the method, we analyzed diseases with highly divergent phenotypes, rather than focusing on a specific subset of phenotypically related diseases. We found that SuMs, and in particular core SuMs, were enriched for GWAS genes. By comparison, no enrichment of GWAS genes was found when analyzing all differentially expressed genes.

To test if core SuMs could be used to find novel genes for functional studies, we analyzed our own gene expression microarray and GWAS data from patients with SAR. This is an optimal model of complex diseases in that it has a clearly defined phenotype that occurs at a given time point each year and the external cause (pollen) and key cell-type (lymphocytes) are known (Extended background in Additional file 2). We identified a SuM and a core SuM, which were reproduced in an independent study. We tested for enrichment of GWAS genes by analyzing a large population-based GWAS. This led to the identification of two novel genes in the core SuM. One of those genes, FGF2, was novel in type 1 allergic inflammation. The relevance of this 


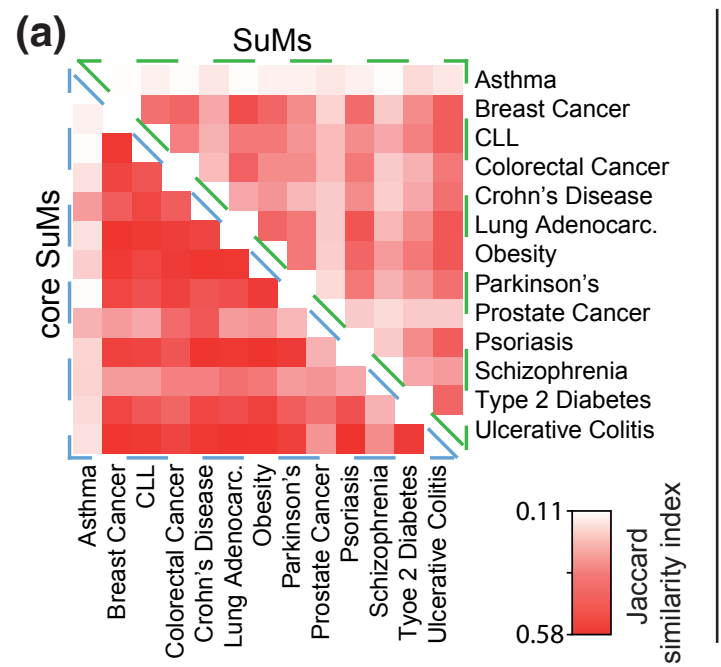

(b)

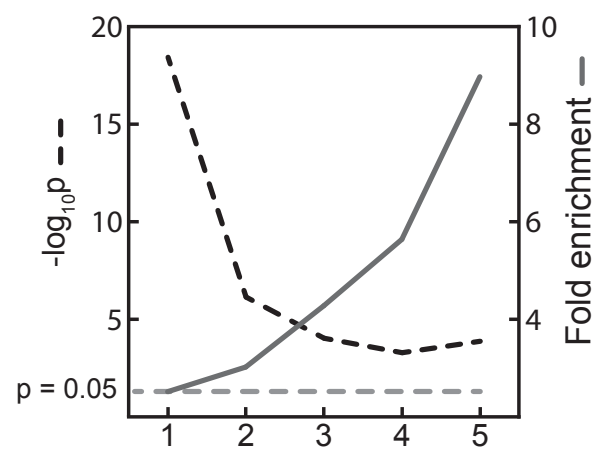

Figure 3 Similarity of core SuMs of different diseases and enrichment of disease genes in the union of core SuMs. (a) Heatmap showing that core SuMs are more similar than SuMs. The color intensity represents similarity, defined by computing the ratio between the number of genes shared by two SuMs and the total number of genes in the two SuMs (Jaccard similarity index). (b) In an extended analysis of GWAS genes of 145 other diseases, the union of the core SuMs was enriched with GWAS genes. This enrichment increased with disease gene pleiotropy. CLL.

gene was supported by transcriptomal analysis following siRNA-mediated knock-down. The knock-down of FGF2 resulted in altered expression of pathways and genes of potential or known relevance for type 1 allergic inflammation. In contrast, we found no enrichment of GWAS genes among all differentially expressed genes. These findings support the hypothesis that core SuMs can be used to identify GWAS genes with moderate effect sizes as well as novel genes for functional studies.

Studies of complex diseases often focus only on specific diseases and the genes associated with them. However, complex diseases show considerable phenotypic and genotypic overlap. Moreover, the effects of individual complex disease genes are generally small, while their collective contribution may be large [33]. This has led to increasing interest in studying groups of diseases and genes $[31,32,34,35]$. In the final part of our study, we made a corresponding change of scale and considered whether core SuMs from the 13 studied diseases were associated with an increased susceptibility for complex diseases, in general. We found that core SuMs tended to overlap, suggesting that they were involved in shared pathogenic mechanisms in complex diseases. This was supported by the union of the core SuMs being enriched for pathways involved in complex diseases. Finally, we tested if the union of the core SuMs was generally enriched for GWAS genes from 145 complex diseases, which represented hundreds of thousands of patients. Indeed, that union was highly enriched for such GWAS genes. Interestingly, the enrichment was greater when considering only GWAS genes associated with more than one disease. Taken together, our findings showed that the core SuMs of highly interconnected disease genes were associated with increased susceptibility for complex diseases.

We propose that the pathways in the core SuMs increase our understanding of how shared pathogenic mechanisms contribute to complex diseases, and also help explain why many of those diseases show phenotypic overlap [36]. From a therapeutic perspective, the core SuMs may be used to prioritize therapeutic novel genes. It should, however, be noted that even though the number of genes in the core SuMs was considerably smaller than all the differentially expressed genes, it may be difficult to find individual target genes. Instead, perhaps drugs targeting combinations of core SuM genes will be required. Another interesting possibility is that the same novel genes might be exploited to develop drugs that target more than one disease. From a diagnostic perspective, different combinations of SuM and core SuM proteins may be useful as diagnostic markers. We propose that such studies can be performed in both specific disease groups and diseases.

A limitation of this study is that it is mainly based on GWAS genes reported in a public database. This may result in missing GWAS genes in some core SuMs, due to too stringent cutoffs. In this study, we analyzed only intragenic SNPs, while intergenic SNPs may have regulatory and disease-causing roles. Another limitation is that the study is based on known physical and functional PPIs. Thus, our results may be confounded by knowledge bias. Moreover, some of the gene expression microarray 
studies used in this effort were performed in tissues that contained mixed cell populations. We anticipate that the increasing accuracy of PPIs as well as availability of gene expression microarray and GWAS data will lead to more accurate identification of SuMs.

\section{Conclusions}

SuMs and core SuMs may be used to find novel genes for functional studies, as well as to increase understanding of the specific and shared pathogenic mechanisms in complex diseases and how they relate to phenotypic manifestations.

\section{Materials and methods}

Definition of susceptibility modules of complex diseases SuMs were defined by integrating PPI network data and differentially expressed genes for each disease. The PPI network was assembled from a large set of functional and physical PPIs obtained from STRING (version 8, using interactions with a confidence score $\geq 0.7$ ). The SuMs were identified using a step-wise process. First, maximal cliques were extracted from the PPI network (A clique is a complete sub-network, that is, a sub-network with links connecting every pair of its nodes (Figure 1a) [37]. Such a clique is maximal if it is not properly contained within another clique.) For this task we employed our custom clique extraction tools as previously applied $[38,39]$. We noted that maximal cliques tend to be highly overlapping and we used all cliques down to a minimum size of 2 that were not part of other cliques. Each clique was tested for enrichment $(P$ $<0.05)$ of differentially expressed genes in the disease using Fisher's exact test (Figure 1b). Differentially expressed genes between the patient and control samples were determined using a Student's $t$-test with a $P<$ 0.05 . Finally, overlapping enriched modules/cliques were mapped onto the PPI network so that each gene was represented only once, and overlapping enriched cliques could be identified as SuMs (Figure 1c). The SuMs included both differentially expressed genes and their neighbors in these cliques. In the SuMs, we identified highly interconnected genes and defined them as core SuMs (Figure 1d). Interconnectivity was measured by calculating the average shortest path length using the Network-Analyzer (v2.6.1) plug-in in Cytoscape (v2.6.0).

\section{Enrichment of core SuMs for GWAS genes}

GWAS genes for all complex diseases were obtained from 'a catalog of published Genome-wide association studies' [40]. We tested for enrichment of GWAS genes using a permutation test. In each permutation, every GWAS gene was replaced with a random gene. The number of random genes present in the corresponding SuMs or core SuMs was noted. This process was repeated 100,000 times. The total number of random genes present in the disease SuMs represented the probability of finding GWAS genes in the SuMs by chance. The significance of the overrepresentation of GWASidentified genes in the SuMs was measured as the ratio of random permutations that included as many or more SuM genes than the true GWAS-identified genes for all 13 diseases. Similarly, enrichment of GWAS genes in the union of core SuMs was also determined for 145 diseases. Pathway enrichment for the union of core SuM genes was determined using Ingenuity Pathway Analysis [17].

\section{Gene-expression microarray analysis in seasonal allergic rhinitis}

Peripheral blood mononuclear cells from patients with SAR and healthy controls were prepared and stimulated with grass pollen extract or diluent for seven days [20]. For gene expression studies, $\mathrm{T}$ helper cells were enriched from the allergen-challenged peripheral blood mononuclear cells using anti-CD4-coated paramagnetic microbeads and a MACS (magnetic cell sorter) system according to the instructions of the manufacturer (Miltenyi Biotec GmbH, Bergisch Gladbach, Germany). cRNA was extracted from 200 ng total RNA using Ambion's Illumina RNA TotalPrep Amplification kit (Ambion, Inc., USA). In vitro transcription reaction and cRNA biotinylation were performed overnight (14 h). The RNA/cRNA concentrations where checked using Nanodrop ND-1000 before and after the amplifications. cRNA quality was controlled by BioRad's Experion electrophoresis station (Bio-Rad Laboratories, Inc., CA, USA). Transcriptional profiling in 12 patients was performed using Illumina's Sentrix ${ }^{\circledR}$ Human-6 Expression BeadChips (Illumina Inc., San Diego, CA, USA) according to the manufacturer's instructions. (The data can be obtained from the Gene Expression Omnibus under accession number GSE18574.) Probes with a detection score below 0.95 were discarded prior to differential expression analysis. Differentially expressed genes were determined using lmFit from the Bioconductor package Limma [41] (Additional file 3). Genes with a P-value < 0.05 after correction for multiple comparisons (false discovery rate) were determined to be differentially expressed. To validate results obtained from the data above, an additional set of patients $(n=3)$ was analyzed on an Affymetrix U133A platform as previously described [20].

\section{GWAS analysis for seasonal allergic rhinitis}

This cohort (NFBC1966) included individuals from the provinces of Oulu and Lapland [42]. In 1997 (when participants were aged 31 years), 8,463 survivors were sent postal questionnaires and invited to clinical examination 
with a $71 \%$ response rate. DNA was collected for the majority of participants and a total of 4,772 individuals were successfully genotyped. All aspects of the study were reviewed and approved by the Ethics Committee of the University of Oulu and participants gave written informed consent. Genotyping was done using the Illumina HumanCNV370-Duo chip. The data were imputed to approximately 2.5 million SNPs using NCBI HapMap II CEU build 35 version 21 after pre-filtering SNPs (genotyping rate $>95 \%, P$-value for HWE deviation $>10^{-4}$, minor allele frequency $>1 \%$, imputation with a confidence call of R2 > 0.5) using IMPUTE [43].

The SAR phenotype was defined as a positive response to the 'have you ever had allergic rhinitis' question in the main questionnaire and a positive skin prick test for grass. Controls had a negative response to the question and a negative skin prick test. A positive skin prick test was defined as a mean wheal reaction to grass extract of at least $3 \mathrm{~mm}$. Participants with a positive reaction to negative control (diluent of allergen extracts) or a negative reaction to positive control $(10 \mathrm{mg} / \mathrm{ml}$ histamine dihydrochloride) were excluded. There were 456 patients that had SAR, while 2,569 individuals were controls.

Association tests for additive effects between SNPs and the defined phenotypes were conducted using the QUICKTEST software and supplemented using R. These analyses were adjusted for sex and relevant principal components of population stratification. Statistically significant SNPs located within and outside the genes of the core SuM were assessed. A SNP was assigned to a gene if that SNP position lies within the start and end region of the gene DNA sequence as defined by the Ensembl database [44]. A higher proportion of SNPs within the genes of the core SuM when compared with the background level of association is indicative of the relevance of the core SuM for the phenotype. The $P$-value of this test is the cumulative probability of finding $k$ or more SNPs in that gene subset when the probability of sampling $k$ SNPs is given by the hypergeometric distribution. The cutoff of the statistical significance of SNP association was determined to be $\alpha$ $=10^{-3}$ via sensitivity analysis of the $P$-values, false discovery rate, true positive rate and odds ratio of the core SuM enrichment (Additional file 6). The false discovery rate was given by $F D R=F P / P$, where $P$ is the number of positive SNP associations and $F P$ is the number of false positive SNP associations within the core SuM under the null hypothesis distribution:

$$
F P=\sum_{x}^{m} p(x, k, m, n) x
$$

where $p(x, k, m, n) x$ is the $P$-value of the hypergeometric distribution when $x$ out of the $k$ significant SNPs are observed within the core SuM, when the core SuM holds $m$ of the total $n$ SNPs. The number of true positive SNP associations $(T P)$ is given by $T P=P-F P$. The odds ratio of the enrichment was defined as the odds of a significant SNP within the core SuM (or any given subset of genes) as compared to the remaining genes. The linkage disequilibrium within the core SuM is assumed to be similar to the expected linkage disequilibrium in the rest of the genome; therefore, GWAS Pvalue distributions within and outside the core SuM should be comparable.

\section{Additional material}

Additional file 1: Additional Table 1 - the genes associated with each disease in the online GWAS catalogue (data obtained on 10 January 2012).

Additional file 2: Additional documentation providing Extended background, Extended experimental methods and Extended results.

Additional file 3: Additional Table 2 - differential expression analysis of allergen-challenged CD4+ cells compared to diluentchallenged controls in SAR.

Additional file 4: Additional Figure 1 - the SuM associated with seasonal allergic rhinitis.

Additional file 5: Additional Table 3 - all disease-associated SNPs in the SAR SuM.

Additional file 6: Additional Figure 2 - sensitivity analysis of the statistical significance level $\alpha$ of a SNP on the Core SuM enrichment.

Additional file 7: Additional Table 4 - differentially expressed genes in Th2 polarized cells following FGF2 knockdown.

Additional file 8: Additional Figure 3 - analysis of FGF2 by siRNAmediated knock-down of FGF2 in Th2 polarized cells, followed by gene expression microarrays.

Additional file 9: Additional Table 5 - pathways enriched in the combined set of core SuM genes.

\section{Abbreviations}

GWAS: genome-wide association studies; PPI: protein-protein interaction; SAR: seasonal allergic rhinitis; siRNA: small interfering RNA; SNP: singlenucleotide polymorphism; SuM: susceptibility module.

\section{Acknowledgements}

We thank Petter Holme for his insightful comments on this manuscript. This work was supported by the European Community's Seventh Framework Programme ([FP7/2007-2012] under grant agreement 223367 (MultiMod)), by the US National Institutes of Health under grants R01-AA-018776 and 3P20MD000516-07S1, and by the Swedish Research Council. SC is an EMBO Long Term Fellow.

\section{Author details}

'The Centre for Individualized Medication, Linköping University Hospital, Linköping University, Linköping, SE-58185, Sweden. ${ }^{2}$ MRC-Laboratory of Molecular Biology, University of Cambridge, Hills Road, Cambridge, CB2 OQH, UK. ${ }^{3}$ Department of Genomics of Common Disease, School of Public Health, Imperial College, London, W2 1PG, UK. ${ }^{4}$ Department of Child and Adolescent Health, National Institute of Health and Welfare, University of Oulu, Oulu, Fl90101, Finland. ${ }^{5}$ Mathematical Sciences, Chalmers University of Technology, University of Gothenburg, Gothenburg, SE-412 96, Sweden. ${ }^{6}$ Department of Electrical Engineering and Computer Science, University of Tennessee, Knoxville, TN 37996-2250, USA. ${ }^{7}$ Respiratory Epidemiology and Public Health 
Group, National Heart and Lung Institute, Imperial College, London, SW7 2AZ, UK. ${ }^{8}$ Unit for Paediatric Allergology, Queen Silvia Children's Hospital, Gothenburg, SE-416 85 Sweden.

\section{Authors' contributions}

$\mathrm{FB}$ and SC were involved in conception, design, acquisition, analysis and interpretation of the data and drafting the manuscript. ALC and LC were involved in conception, design, acquisition, analysis and interpretation of the data. MRJ, RJ and MAL were involved in conception, design and interpretation of the data. AR, GR and HW were involved in acquisition and analysis of the data. MB supervised the entire study and was involved in conception, design, interpretation of the data and drafting the manuscript. All authors were involved in critically revising the manuscript for intellectual content and have given final approval of this version to be published.

\section{Competing interests}

The authors declare that they have no competing interests.

Received: 27 February 2012 Revised: 25 May 2012

Accepted: 15 June 2012 Published: 15 June 2012

\section{References}

1. Ge H, Liu Z, Church GM, Vidal M: Correlation between transcriptome and interactome mapping data from Saccharomyces cerevisiae. Nat Genet 2001, 29:482-486.

2. Minguez P, Dopazo J: Assessing the biological significance of gene expression signatures and co-expression modules by studying their network properties. PLoS One 2011, 6:e17474.

3. Narayanan M, Vetta A, Schadt EE, Zhu J: Simultaneous clustering of multiple gene expression and physical interaction datasets. PLoS Comput Biol 2010, 6:e1000742.

4. Ramani AK, Li Z, Hart GT, Carlson MW, Boutz DR, Marcotte EM: A map of human protein interactions derived from co-expression of human mRNAs and their orthologs. Mol Syst Biol 2008, 4:180.

5. Chen J, Liang H, Fernandez A: Protein structure protection commits gene expression patterns. Genome Biol 2008, 9:R107.

6. Pujana MA, Han JD, Starita LM, Stevens KN, Tewari M, Ahn JS, Rennert G, Moreno V, Kirchhoff T, Gold B, Assmann V, Elshamy WM, Rual JF, Levine D, Rozek LS, Gelman RS, Gunsalus KC, Greenberg RA, Sobhian B, Bertin N, Venkatesan K, Ayivi-Guedehoussou N, Sole X, Hernandez P, Lazaro C, Nathanson KL, Weber BL, Cusick ME, Hill DE, Offit K, et al: Network modeling links breast cancer susceptibility and centrosome dysfunction. Nat Genet 2007, 39:1338-1349.

7. Barabasi AL, Gulbahce N, Loscalzo J: Network medicine: a network-based approach to human disease. Nat Rev Genet 2011, 12:56-68.

8. Goldstein DB: Common genetic variation and human traits. N Engl J Med 2009, 360:1696-1698

9. Schadt EE: Molecular networks as sensors and drivers of common human diseases. Nature 2009, 461:218-223.

10. Lage K, Karlberg EO, Storling ZM, Olason PI, Pedersen AG, Rigina O, Hinsby AM, Tumer Z, Pociot F, Tommerup N, Moreau Y, Brunak S: A human phenome-interactome network of protein complexes implicated in genetic disorders. Nat Biotechnol 2007, 25:309-316.

11. Goh Kl, Cusick ME, Valle D, Childs B, Vidal M, Barabasi AL: The human disease network. Proc Natl Acad Sci USA 2007, 104:8685-8690.

12. Oti M, Snel B, Huynen MA, Brunner HG: Predicting disease genes using protein-protein interactions. J Med Genet 2006, 43:691-698.

13. Feldman I, Rzhetsky A, Vitkup D: Network properties of genes harboring inherited disease mutations. Proc Natl Acad Sci USA 2008, 105:4323-4328.

14. Barrenas F, Chavali S, Holme P, Mobini R, Benson M: Network properties of complex human disease genes identified through genome-wide association studies. PLoS One 2009, 4:e8090.

15. Chuang HY, Lee E, Liu YT, Lee D, Ideker T: Network-based classification of breast cancer metastasis. Mol Syst Biol 2007, 3:140.

16. Woodfolk JA: T-cell responses to allergens. J Allergy Clin Immunol 2007, 119:280-294, quiz 295-286.

17. Bruhn S, Barrenas F, Mobini R, Andersson BA, Chavali S, Egan BS, Hovig E, Sandve GK, Langston MA, Rogers G, Wang H, Benson M: Increased expression of IRF4 and ETS1 in CD4(+) cells from patients with intermittent allergic rhinitis. Allergy 2012, 67:33-40.
18. Banfield G, Watanabe H, Scadding G, Jacobson MR, Till SJ, Hall DA, Robinson DS, Lloyd CM, Nouri-Aria KT, Durham SR: CC chemokine receptor 4 (CCR4) in human allergen-induced late nasal responses. Allergy 2010, 65:1126-1133.

19. Benson M, Langston MA, Adner M, Andersson B, Torinssson-Naluai A, Cardell LO: A network-based analysis of the late-phase reaction of the skin. J Allergy Clin Immunol 2006, 118:220-225.

20. Benson M, Carlsson L, Guillot $G$, Jernas M, Langston MA, Rudemo M, Andersson B: A network-based analysis of allergen-challenged CD4+ T cells from patients with allergic rhinitis. Genes Immun 2006, 7:514-521.

21. Ramasamy A, Curjuric I, Coin L, Kumar A, McArdle WL, Imboden M, Leynaert B, Kogevinas M, Schmid-Grendelmeier P, Pekkanen J, Wjst M, Bircher AJ, Sovio U, Rochat T, Hartikainen AL, Balding DJ, Jarvelin MR, Probst-Hensch N, Strachan DP, Jarvis DL: A genome-wide meta-analysis of genetic variants associated with allergic rhinitis and grass sensitization and their interaction with birth order. J Allergy Clin Immunol 2011, 128:996-1005.

22. Dong C, Yang DD, Tournier C, Whitmarsh AJ, Xu J, Davis RJ, Flavell RA: JNK is required for effector T-cell function but not for T-cell activation. Nature 2000, 405:91-94.

23. Dong C, Yang DD, Wysk M, Whitmarsh AJ, Davis RJ, Flavell RA: Defective T cell differentiation in the absence of Jnk1. Science 1998, 282:2092-2095.

24. Chu X, Ci X, He J, Wei M, Yang X, Cao Q, Li H, Guan S, Deng Y, Pang D, Deng $X$ : A novel anti-inflammatory role for ginkgolide $B$ in asthma via inhibition of the ERK/MAPK signaling pathway. Molecules 2011, 16:7634-7648.

25. Hashizume $H$, Hamalainen $H$, Sun Q, Sucharczuk A, Lahesmaa R: Downregulation of mafB expression in T-helper cells during early differentiation in vitro. Scand J Immunol 2003, 57:28-34.

26. Chen Q, Dowhan DH, Liang D, Moore DD, Overbeek PA: CREB-binding protein/p300 co-activation of crystallin gene expression. J Biol Chem 2002, 277:24081-24089.

27. Cornejo-Garcia JA, Fernandez TD, Torres MJ, Carballo M, Hernan I, Antunez C, Blanca M, Mayorga C: Differential cytokine and transcription factor expression in patients with allergic reactions to drugs. Allergy 2007, 62:1429-1438.

28. Erpenbeck VJ, Hagenberg A, Krentel H, Discher M, Braun A, Hohlfeld JM, Krug N: Regulation of GATA-3, c-maf and T-bet mRNA expression in bronchoalveolar lavage cells and bronchial biopsies after segmental allergen challenge. Int Arch Allergy Immunol 2006, 139:306-316.

29. Tenda Y, Yamashita M, Kimura MY, Hasegawa A, Shimizu C, Kitajima M, Onodera A, Suzuki A, Seki N, Nakayama T: Hyperresponsive TH2 cells with enhanced nuclear factor-kappa B activation induce atopic dermatitis-like skin lesions in Nishiki-nezumi Cinnamon/Nagoya mice. J Allergy Clin Immunol 2006, 118:725-733.

30. El-Hashim AZ, Renno WM, Abduo HT, Jaffal SM, Akhtar S, Benter IF: Effect of inhibition of the ubiquitin-proteasome-system and IkappaB kinase on airway inflammation and hyperresponsiveness in a murine model of asthma. Int J Immunopathol Pharmacol 2011, 24:33-42.

31. Ramos PS, Criswell LA, Moser KL, Comeau ME, Williams AH, Pajewski NM, Chung SA, Graham RR, Zidovetzki R, Kelly JA, Kaufman KM, Jacob CO, Vyse TJ, Tsao BP, Kimberly RP, Gaffney PM, Alarcon-Riquelme ME, Harley JB, Langefeld CD: A Comprehensive analysis of shared loci between systemic lupus erythematosus (SLE) and sixteen autoimmune diseases reveals limited genetic overlap. PLoS Genet 2011, 7:e1002406.

32. Sivakumaran S, Agakov F, Theodoratou E, Prendergast JG, Zgaga L, Manolio T, Rudan I, McKeigue P, Wilson JF, Campbell H: Abundant pleiotropy in human complex diseases and traits. Am J Hum Genet 2011, 89:607-618.

33. Petretto E, Liu ET, Aitman TJ: A gene harvest revealing the archeology and complexity of human disease. Nat Genet 2007, 39:1299-1301.

34. Sirota M, Schaub MA, Batzoglou S, Robinson WH, Butte AJ: Autoimmune disease classification by inverse association with SNP alleles. PLoS Genet 2009, 5:e1000792.

35. Torkamani A, Dean B, Schork NJ, Thomas EA: Coexpression network analysis of neural tissue reveals perturbations in developmental processes in schizophrenia. Genome Res 2010, 20:403-412.

36. Chavali S, Barrenas F, Kanduri K, Benson M: Network properties of human disease genes with pleiotropic effects. BMC Syst Biol 2010, 4:78. 
37. Bomze J, Budinich M, Pardalos P, Pelillo M: The maximum clique problem. In Handbook of Combinatorial Optimization. Volume 4. Edited by: Du D-Z, Pardalos PM. Kluwer Academic Publishers; 1999.

38. Chesler EJ, Lu L, Shou S, Qu Y, Gu J, Wang J, Hsu HC, Mountz JD, Baldwin NE, Langston MA, Threadgill DW, Manly KF, Williams RW: Complex trait analysis of gene expression uncovers polygenic and pleiotropic networks that modulate nervous system function. Nat Genet 2005, 37:233-242.

39. Voy BH, Scharff JA, Perkins AD, Saxton AM, Borate B, Chesler EJ, Branstetter LK, Langston MA: Extracting gene networks for low-dose radiation using graph theoretical algorithms. PLoS Comput Biol 2006, 2: e89.

40. Hindorff LA, Sethupathy P, Junkins HA, Ramos EM, Mehta JP, Collins FS, Manolio TA: Potential etiologic and functional implications of genomewide association loci for human diseases and traits. Proc Natl Acad Sci USA 2009, 106:9362-9367.

41. Smyth GK: Linear models and empirical bayes methods for assessing differential expression in microarray experiments. Stat Appl Genet Mol Biol 2004, 3:Article3.

42. Rantakallio $P$, Leino $S$ : Social and biological factors adding to the risks of infants. Katilolehti 1969, 74:520-522.

43. Marchini J, Howie B, Myers S, McVean G, Donnelly P: A new multipoint method for genome-wide association studies by imputation of genotypes. Nat Genet 2007, 39:906-913.

44. Hubbard T, Andrews D, Caccamo M, Cameron G, Chen Y, Clamp M, Clarke L, Coates G, Cox T, Cunningham F, Curwen V, Cutts T, Down T, Durbin R, Fernandez-Suarez XM, Gilbert J, Hammond M, Herrero J, Hotz H, Howe K, lyer V, Jekosch K, Kahari A, Kasprzyk A, Keefe D, Keenan S, Kokocinsci F, London D, Longden I, McVicker G, et al: Ensembl 2005. Nucleic Acids Res 2005, 33:D447-453.

doi:10.1186/gb-2012-13-6-r46

Cite this article as: Barrenäs et al:: Highly interconnected genes in disease-specific networks are enriched for disease-associated polymorphisms. Genome Biology 2012 13:R46.

\section{Submit your next manuscript to BioMed Central} and take full advantage of:

- Convenient online submission

- Thorough peer review

- No space constraints or color figure charges

- Immediate publication on acceptance

- Inclusion in PubMed, CAS, Scopus and Google Scholar

- Research which is freely available for redistribution

Submit your manuscript at www.biomedcentral.com/submit
Biomed Central 